\title{
Identification in static luminance and color noise
}

\author{
Piet Bijl, Marcel P. Lucassen \& Jolanda Roelofsen \\ TNO Human Factors \\ P.O. Box 23 \\ 3769 ZG Soesterberg \\ The Netherlands \\ Phone: +31 346356 277, fax: +31 346353977 \\ e-mail: piet.bij1@tno.nl, marcel.lucassen@tno.nl
}

\begin{abstract}
If images from multiple sources (e.g. from the different bands of a multi-band sensor) are displayed in color, Signal and Noise may appear as luminance and color differences in the image. As a consequence, the perception of color differences may be important for Target Acquisition performance with fused imagery. Luminance and color can be represented in a 3-D space; in the CIE 1994 color difference model, the three perceptual directions are lightness (L*), chroma $\left(\mathrm{C}^{*}\right)$ and hue $\left(\mathrm{h}^{*}\right)$. In this 3 -D color space, we performed two perception experiments. In Experiment 1 , we measured human observer detection thresholds (JND's) for uniformly distributed static noise (fixed pattern noise) in $\mathrm{L}^{*}$, $\mathrm{C}^{*}$ or $\mathrm{h}^{*}$ on a uniform background. The results show that the JND for noise in $\mathrm{L}^{*}$ is significantly lower than for noise in $\mathrm{C}^{*}$ or $\mathrm{h}^{*}$. In Experiment 2, we measured the threshold contrast for identification (orientation discrimination) of a Ushaped test target on a noisy background. With test symbol and background noise in $\mathrm{L}^{*}$, the ratio between signal threshold and noise level is constant. With the symbol in a different direction, we found little dependency on noise level. The results may be used to optimize the use of color to human detection and identification performance with multi-band systems.
\end{abstract}

Keywords: Target Acquisition, detection, identification, color, noise, multi-band, human observer

\section{INTRODUCTION}

Until now, thermal IR image forming systems are usually single- or broad-band, i.e. they have one channel with a spectral sensitivity in the Mid Wave (MW, 3-5 $\mu \mathrm{m}$ ) or Long Wave (LW, 8-12 $\mu \mathrm{m}$ ) infrared band. These systems usually produce a grayscale image that is related to the amount of radiation emitted (or reflected) by the scene in the spectral region of the sensor. If two objects produce the same amount of radiance on the Focal Plane Array in the spectral region of the sensor, this will result in the same detector output.

The emergence of multi-band sensor technology, e.g. in the thermal infrared, promises significant improvements in military task performance. With these new sensor systems, targets may be distinguished not only on the basis of differences in radiation magnitude, but also on differences in spectral properties. This opens the way to alternative presentation methods, for example the use of color to present these spectral differences. The additional information may improve, for instance: 1) Target Acquisition (TA) performance (search, detection recognition, and identification), 2) Situational Awareness (SA), 3) discrimination between military and civilian targets, 4) discrimination between real targets and decoys, 5) automatic detection of point targets or camouflaged targets, 6) automatic or aided target recognition, 6) operability in a wider range of climates and weather conditions, and/or 7) viewing comfort.

The new technologies, however, also raise new research questions. Until now, nobody really knows the potential of multi-band systems, and how to make optimal use of the new technology. At present, international task groups (e.g. NATO TG.33) address questions such as: 1) which spectral bands provide the most useful information, and 2) how can this information be presented to a human observer in the best way? Most likely, the answers are task and scenario dependent. In order to have optimal detection performance, a scheme may be used that maximizes the contrast or the 
conspicuity of a target. However, if SA has priority, the best way to present multi-band IR imagery to a human observer may be using the Color the Night algorithm developed by Toet ${ }^{1}$, yielding natural colors.

A specific problem is that each channel of a multi-band system provides signal and noise. If the information of the channels is combined and presented to a human observer in color, the resulting image contains color noise. Depending on the combination of the channels the resulting noise may be more or less disturbing and threshold signal-to-noise ratios may be different. In order to optimize the use of multi-band imaging systems, it is essential to get insight in the ability of a human observer to perceive colored noise and targets in colored noise. Although in color science welldefined metrics are available for specification of (differences between) colors that are spatially uniform, there are no such tools for colors that are not uniform, such as spatial noise. One of the first to address this was Driggers ${ }^{2}$, who investigated the detection of simple color targets in a noisy color background.

In the present study, we systematically measure in color space 1) detection thresholds for different types of static colored noise (in lightness, chromaticity or hue) on a uniform background, and 2) threshold contrasts (in lightness, chromaticity or hue) for identification (orientation discrimination) of a U-shaped test target on a noisy background. The experiments are an extension of earlier work by Lucassen $\&$ Bijl $^{3}$. The results may be used to 1) avoid disturbance of the image by noise, and 2) maximize the identification of a test target in the presence of noise.

In Chapter 2, the theoretical background is provided. Methods are described in Chapter 3. The results of the experiments are given in Chapter 4, and the results discussed in Chapter 5.

\section{THEORETICAL BACKGROUND}

Under daylight conditions the Human Visual System is able to perceive color due to three types of cones with different spectral sensitivities: L (Long wave sensitive), M (Middle wave sensitive) and S (Short wave sensitive). Since the color (including luminance) coding is limited to three numbers (the responses of the three types of cones), all colors can be represented in a 3-D color space or color chromaticity diagram. Several representations of color space exist, e.g. CIE (Commission Internationale de l'Eclairage) $X Y Z, L * a * b *$ or $L^{*} C^{*} h^{*}{ }^{4}$.

Colors are often ordered along the three perceptual directions Lightness, Chroma, and Hue, denoted by the symbols $L^{*}$, $C^{*}$ and $h^{*}$ (Fig. 1). Lightness classifies a color according to a series of greys ranging from black $\left(L^{*}=0\right)$ to white $\left(L^{*}=100\right)$ and refers to a light level that is perceived relative to white. Chroma describes the degree of colorfulness (saturation), i.e. the difference between the color and the neutral grey of that same lightness. Hue is what we call the 'color' of an object: red, yellow, blue, etc. and classifies the color as an angle, in degrees $\left(0-360^{\circ}\right)$, around a color wheel. Using these three attributes a color is accurately identified and can be distinguished from any other.

Using the CIE $1994\left(\Delta L^{*} \Delta C^{*} \Delta H^{*}\right)$ color-difference model, we can express the strength of the noise component and of the test pattern as the total color-difference $\Delta E^{*}{ }_{94}$ with the uniform background using the following equation ${ }^{5}$ :

$$
\Delta E *{ }_{94}=\sqrt{\left(\frac{\Delta L *}{k_{L} S_{L}}\right)^{2}+\left(\frac{\Delta C *}{k_{C} S_{C}}\right)^{2}+\left(\frac{\Delta H *}{k_{H} S_{H}}\right)^{2}}
$$

$\Delta E^{*}{ }_{94}$ is built up from three components: the distance between two color samples in lightness $\left(\Delta L^{*}\right)$, in chroma $\left(\Delta C^{*}\right)$, and in hue $\left(\Delta H^{*}\right)$. These distances are weighted with functions $S_{L}, S_{C}, S_{H}{ }^{5}$ and parametric factors, $k_{L}, k_{C}, k_{H}$. The kfactors have a value of 1 under CIE94 reference conditions, and they are used to adjust the relative weighting of the lightness, chroma, and hue components for various viewing conditions and applications that differ from the reference conditions. Although there is a more recent color difference formula ${ }^{6}$ we deliberately work with the 1994 version, mainly because of the symmetry in the latter and practical issues related to implementation of the formula into our software for generating the stimuli. 


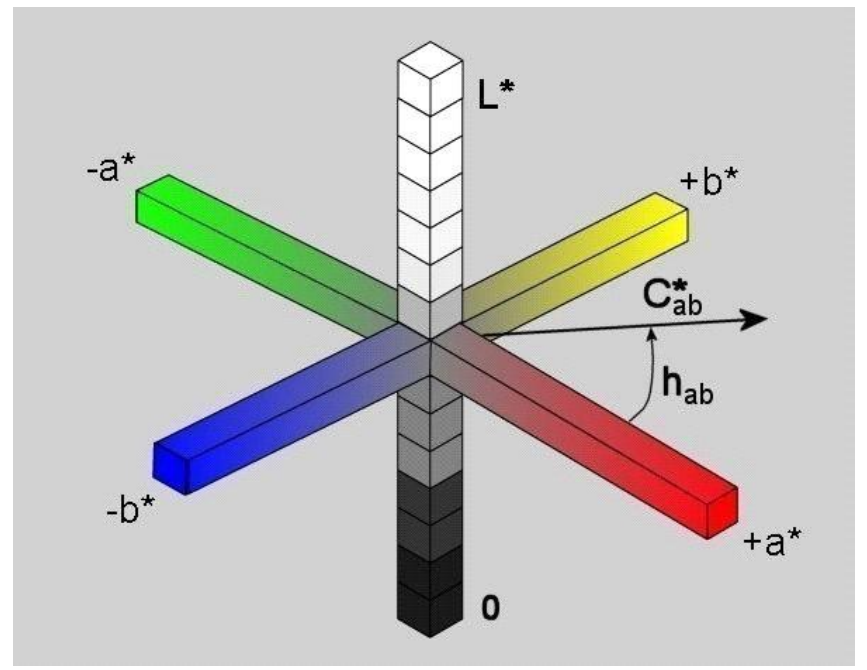

Fig. 1: The three perceptual directions of color: Lightness $\left(\mathrm{L}^{*}\right)$, Chroma $\left(\mathrm{C}^{*}\right)$ and Hue (h*). (figure adapted from unknown source).

In Experiment 1, the total color-difference of static noise will be determined at the threshold level. Since we deviate from the CIE94 reference conditions, we cannot expect the k-factors to be equal to 1 . Threshold was determined for noise in the three directions, $L^{*}, C^{*}$, and $h^{*}$ separately. When selecting one direction, the other two remain unchanged, which simplifies equation (1) to the following three cases:

$$
\begin{array}{ll}
\Delta E *_{94}=\left(\frac{\Delta L^{*}}{k_{L} S_{L}}\right) & \Delta C^{*}=\Delta H^{*}=0 \\
\Delta E *_{94}^{*}=\left(\frac{\Delta C *}{k_{C} S_{C}}\right) & \Delta L^{*}=\Delta H^{*}=0 \\
\Delta E *_{94}=\left(\frac{\Delta H *}{k_{H} S_{H}}\right) & \Delta L^{*}=\Delta C^{*}=0
\end{array}
$$

After Experiment 1, the parametric factors $k_{L}, k_{C}$, and $k_{H}$ were assigned the threshold values for the noise, so that the threshold corresponds to $\Delta E^{*}{ }_{94}=1$.

In Experiment 2, the visibility of a test symbol (a square U-shaped symbol) in the presence of different amounts of $L^{*}$ noise was studied. The test symbol could be addressed in lightness $\left(L^{*}\right)$, chroma $\left(C^{*}\right)$ or hue $\left(h^{*}\right)$. It was expected that when these directions are the same (for test symbol and noise), it would become more difficult to see the stimulus when the noise is increasing. However, the noise level should not have an influence on the perception of the test symbol when the directions are different. 


\section{METHODS}

\section{1}

\section{EXPERIMENTAL SET-UP}

The experiments were carried out in a darkened room. Subjects were seated at a $50 \mathrm{~cm}$ distance from a 18.1-inch color display. Before each session a calibration was performed. The monitor was set at maximum luminance of $120 \mathrm{~cd} / \mathrm{m}^{2}, \mathrm{a}$ white point of $6500 \mathrm{~K}$, and gamma 2.2 (for each of the three primary channels).

\subsection{STIMULI}

Examples of the stimuli used in Experiments 1 and 2 are shown in Fig. 2 (left and right image, respectively). Each display consisted of four quadrants, one of which contained the test stimulus (to be detected by the observers). With each new stimulus presentation the test stimulus was randomly assigned to one of the four quadrants. The quadrants were in turn subdivided in smaller squares $\left(0.58^{\circ}\right.$ each $)$ that could be individually addressed with a color specification in terms of $L^{*}, C^{*}$ and $h^{*}$ (i.e. fixed pattern noise).
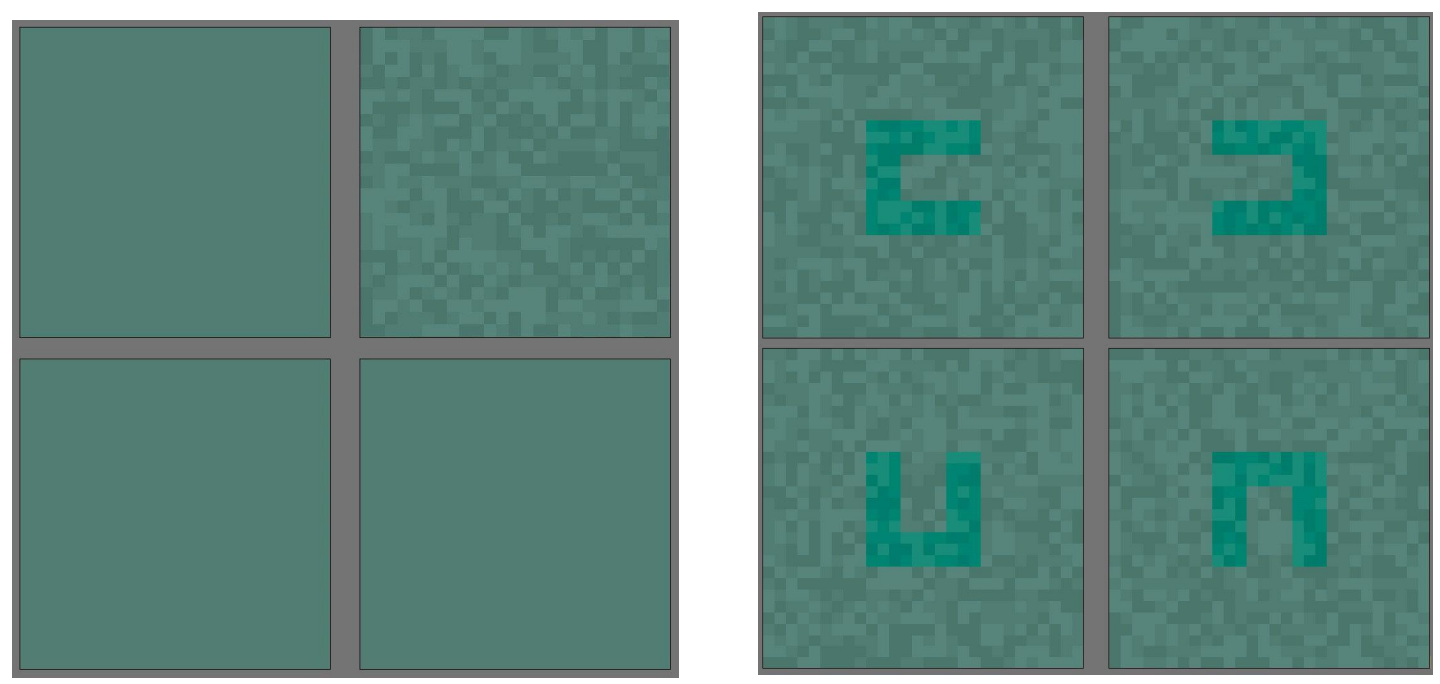

Fig. 2: Examples of the stimuli used in Experiment 1 (left) and Experiment 2 (right). The background color is at $L^{*}=50, C^{*}=20, h^{*}=180$, and the background noise is in direction $L^{*}$. The additional U-shaped symbol on the right is incremental in the direction $C^{*}$ (higher saturation). Subjects had to look for the test stimulus, being the quadrant containing noise in Experiment 1 or the quadrant containing the symbol in up-orientation in Experiment 2.

In Experiment 1 the quadrants were made up of $625(25 \times 25)$ smaller squares, showing a common colored background and one quadrant contained additional noise (see Fig. 2, left side). Each quadrant subtends a visual angle of $14.5^{\circ}$ at 0.50 $\mathrm{m}$ distance. The amount of noise was set by the value of $\Delta E^{*}{ }_{94}$ and under control of a staircase procedure (to be explained in the next section). The noise could be applied in one of the three perceptual directions $L^{*}, C^{*}$ or $h^{*}$, but not simultaneously in two or three. Each small square was randomly assigned $+1,0$ or -1 times the noise level specified by $\Delta E^{*}{ }_{94}$. What this means in terms of $L^{*}, C^{*}$ and $h^{*}$ depends on the selected noise direction and the values for the scaling factors $\left(k\right.$ and $S$ ) in equations (2)-(4). In any case, the scene-averaged $L^{*}, C^{*}, h^{*}$ values of the quadrant with noise are identical to those of the quadrants without noise. 
In Experiment 2 the quadrants were made up of 784 (28x28) small squares, showing a colored background with noise and an additional test symbol with one of four possible orientations (Up, Down, Left, Right). Each quadrant subtends a visual angle of $16.2^{\circ}$ at $0.50 \mathrm{~m}$. All quadrants contained a different symbol orientation. Subjects had to look for the quadrant containing the symbol in the up-orientation. The test symbol was about one-third the size of the quadrant, and incremental to the background noise.

\subsection{PSYCHOPHYSICAL MEASUREMENT PROCEDURE}

A four alternative forced-choice (4AFC) procedure was implemented in a staircase method ${ }^{8,9}$. Each stimulus presentation consisted of four quadrants. Subjects had to indicate the quadrant that - according to their perception contained the test stimulus. This was done by pressing the space bar of the keyboard, which moved a marker from one quadrant to the next, and the enter-key was pressed as a confirmation. After a wrong response the subjects heard a beep. This feedback makes the observers feel sure and has a stabilizing effect on their responses ${ }^{8}$. The procedure yields approximately $75 \%$ correct answers.

There was no time restriction and subjects could work at their own speed. One trial took about 3 minutes in Experiment 1 and about 5 minutes in Experiment 2. Experimental sessions lasted for about one hour.

\subsection{SUBJECTS}

Three subjects participated in this series of experiments, one male and two females, age ranging from 23 to 39 . They all had normal color vision as confirmed by different color vision tests (Ishihara, Farnsworth D15 and TNO VisionScreener ${ }^{13}$ ). All subjects were familiar with the purpose of the experiments.

\section{RESULTS}

In Experiment 1, noise thresholds were measured on uniform backgrounds with $L^{*}=50, C^{*}=20$, and with hue axis sampled at $30^{\circ}$ intervals. An example of the test stimulus is shown in Fig. 2 (left graph). For all hue values we found that threshold level for the detection of noise in $L^{*}$ is much smaller than for the other two directions. Thus, the parametric factors $k_{L}, k_{C}$, and $k_{H}$ are different for $\mathrm{L}^{*}, \mathrm{C}^{*}$ and $\mathrm{h}^{*}$ and deviate from 1 (the value for the CIE94 reference conditions).

Before Experiment 2 was started, the parametric factors $k_{L}, k_{C}, k_{H}$ in equations (2)-(4), were set equal to the threshold values obtained in Experiment 1 (these are different for each observer and each hue value). This scaling has two advantages. First, $\Delta E^{*}{ }_{94}=1$ now corresponds to $1 \mathrm{JND}$ (just noticeable difference) of the noise ${ }^{1}$. This greatly simplifies the interpretation of the results of Experiment 2. Second, as long as the noise does not simultaneously address two or three of the perceptual directions, $1 \mathrm{JND}$ in $L^{*}$ also corresponds to $1 \mathrm{JND}$ in $C^{*}$ and to $1 \mathrm{JND}$ in $h^{*}$.

In Experiment 2, thresholds were measured for correctly judging the orientation of a U-shaped test symbol that was superimposed (incremental) on background $L^{*}$ noise; see the example in Fig. 2, right graph. Background noise levels were $\Delta E^{*}{ }_{94}=0,5,10,15$ and 20, respectively. Background color was $L^{*}=50, C^{*}=20$ and $h^{*}=0^{\circ}$.

The results of the Experiment are shown in Fig. 3 for observer JR. Plotted is the threshold as a function of the amount of background noise. Filled diamonds: target in $L^{*}$; Open squares: target in $C^{*}$; open triangles: $\operatorname{target}$ in $h^{*}$.

In Fig. 3, we see that the threshold for a target symbol in $L^{*}$ increases linearly with noise. This means that the SNR (signal-to-noise ratio) is approximately constant over this range. For this condition, $S N R \approx 1$. Thresholds for a target in $C^{*}$ or $h^{*}$ are independent of the amount of noise in $L^{*}$. Thus, the visibility of a colored symbol is extremely insensitive to fixed pattern noise in luminance.

1 Here we define 1 JND as the $75 \%$ correct threshold 


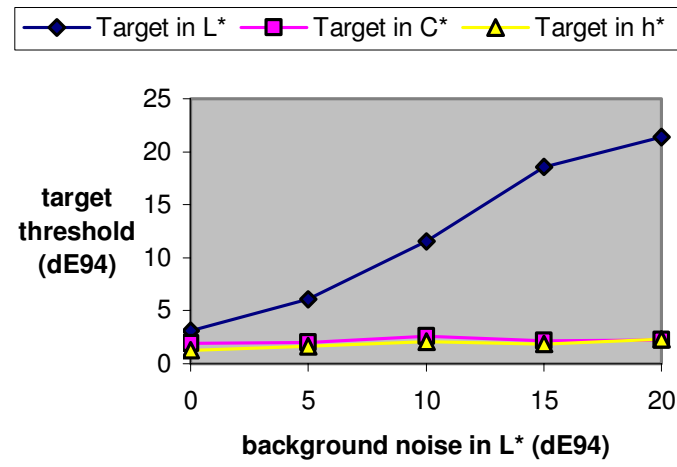

Fig. 3: Results of Experiment 2. With noise in $L^{*}$, threshold for a target symbol in $L^{*}$ increases linearly but thresholds for a target in $C^{*}$ or $h^{*}$ are independent of the amount of noise in $\mathrm{L}^{*}$.

\section{DISCUSSION}

In Experiment 1, we found that that threshold values for the detection of noise in direction $L^{*}$ is considerably smaller than that for the directions $C^{*}$ and $h^{*}$. This means that the values for the CIE reference condition cannot be applied to the noise thresholds and need to be adapted in order to be able to predict the visibility of color noise in sensor systems.

In Experiment 2, we found that identification thresholds for symbols in $L^{*}$ depend strongly on the amount of noise in $L^{*}$, but that symbols in $C^{*}$ or $h^{*}$ are not affected by such noise. This is predicted by the CIE94 color difference equation (1).

\section{Practical implications}

The channels from a multi-band sensor are often simply combined into a color image using a false-coloring algorithm. However, most probably this is rarely the optimum choice for the task and scenario at hand. For instance, in order to obtain maximum Situation Awareness, one may strive for a natural reproduction so that the colors of objects have their natural color ${ }^{1}$. Another possibility is to strive for a color reproduction that enhances differences in the individual sensor images as much as possible. An alternative that is offered by the results of our study is that, depending on the amount of noise in the separate images, these are combined in a color image in such a way that the detection or identification thresholds for objects are as low as possible. The optimal choice may depend on many factors, such as the size of the target or the SNR in the different channels, and these may have to be based on prior knowledge or expectations. One may consider a multiple or switchable display system that is able to present different choices for different tasks (e.g. Situation Awareness and maximum detection) or applications.

Another area of application is in the removal of color noise in digital images. Small variations in color can be removed as long as they are well below the detection threshold, which may finally lead to more effective image compressions. The success of such noise filtering is dependent on the angular size of the noise elements (both the size and viewing distance are relevant here). 


\section{Future research}

In this study, we have varied only a limited amount of parameters. For instance, we have not yet studied the effect of more than one noise component at the same time. Further, the effect of size may play an important role. After a more thorough investigation of these factors, the following steps may lead to a firmly-based framework for the efficient use of color in multi-band imaging systems:

- Detection of noise in real color imagery

- Search, detection and identification of targets in noise in real color imagery

- Description of the optimum color representation scheme, depending on task, target and background spectral properties, target size, etc.

- Development of a color Target Acquisition model

- Validation of the multi-band test method proposed by Bijl \& Hogervorst ${ }^{11}$, an extension of the TOD test method ${ }^{12}$ for multi-spectral imaging sensors.

\section{REFERENCES}

1. Toet, A. (2003). Natural color mapping for multiband nightvision imagery. Information Fusion 4, 155-166.

2. Driggers, R.G., Krapels, K., Vollmerhausen, R., Warren, P., Scribner, D., Howard, G., Tsou, B.H. \& Krebs, W.K. (2001). Target detection threshold in noisy color imagery. SPIE Proceedings Vol. 4372, 162-169.

3. Lucassen, M.P., Bijl, P. (2004). The CIE94 Color Difference Formula for Describing Visual Detection Thresholds in Static Noise. Second European Conference on Color in Graphics, Imaging and Vision (CGIV 2004), Aachen, Germany; April 2004; p. 8-11; ISBN / ISSN: 0-89208-250-X

4. Wyszecki, G. \& Stiles, W.S. (1982). Color Science, Concepts and Methods, Quantitative Data and Formulae, 2 nd. Edition, New York: Wiley.

5. Fairchild, M.D. (1998). Color appearance models. Reading, Massachusetts, Addison-Wesley.

6. Luo, M.R. \& Rigg, G.C.B. (2001). The development of the CIE 2000 color-difference formula: CIEDE2000. Color Research and Application, 26, 5, 340-350.

7. Mulligan, J.B., and Ahumada, A.J. Jr., (1992), "Principle methods for color dithering based on models of the human visual system." SID Int. Symp. Dig. Tech. Papers, 23, 194- 197.

8. Bijl, P., Toet, A., \& Valeton, J.M. (2003). Psychophysics and psychophysical measurement procedures. Encyclopedia of Optical Engeneering, Volume, 2176-2187.

9. Bijl, P., Koenderink, J.J., \& Toet, A. (1989). Visibility of blobs with a gaussian luminance profile. Vision Research, 4, 447-456.

10. Roelofsen, J. (2005). The perception of static colored noise. Master's Thesis. University of Amsterdam, Amsterdam, The Netherlands

11. Bijl, P. \& Hogervorst, M.A. (2003). A test method for multi-band imaging sensors. SPIE Proceedings Vol. 5076, 208-219.

12. Bijl, P.\& Valeton, J.M. (1998). TOD, the alternative to MRTD and MRC. Optical Engineering 37, 7, 1976 - 1983.

13. Walraven, J. Lucassen, M.P., Alferdinck, J.W.A.M. , Kooi, F.L. (2002). Automated visual screening; development of computerised vision tests for the TNO VisionScreener. TNO-report TM-02-A044 (Company Confidential). 